\title{
Three-dimensional information from two-dimensional scans: a scanning microscope with postacquisition refocusing capability: supplementary material
}

\author{
Alexander Jesacher ${ }^{1}{ }^{*}$, Monika Ritsch-Marte $^{1}$, And Rafael Piestun ${ }^{1,2}$ \\ ${ }^{1}$ Division of Biomedical Physics, Innsbruck Medical University, Müllerstraße 44, 6020 Innsbruck, Austria \\ ${ }^{2}$ Department of Electrical and Computer Engineering, University of Colorado, Boulder, CO 80309, USA \\ *Corresponding author: alexander.jesacher@i-med.ac.at
}

Published 27 February 2015

\begin{abstract}
This document provides supplementary information to "Three-dimensional information from twodimensional scans: a scanning microscope with postacquisition refocusing capability," http:// dx.doi.org/10.1364/optica.2.000210. It contains detailed explanations of the data processing steps required for RESCH. (C) 2015 Optical Society of America
\end{abstract}

http://dx.doi.org/10.1364/optica.2.000210.s001

\section{DATA ACQUISITION AND PROCESSING}

RESCH is a camera-based point scanning microscopy technique in the sense that an image of the sample response to laser exposure is taken sequentially at every single sampling point $(i, j)$ in the specimen. The size of these images can be quite small, about $25 \times 25$ pixels are typically sufficient. Figure S1a shows a table containing exemplary raw images $R_{i, j}(x, y)$. They are parts of the raw data from an imaging experiment with stained microtubule networks in fixed African green monkey kidney cells. A larger part of the raw data is provided in the form of a movie in Media S1. The movie frame rate is smaller by a factor of approximately three compared to the original data acquisition rate. The complete raw data of this experiment consist of 10,000 such images, each showing a distorted double-helix pattern whose intensity, position and rotational angle depend on the local structure of the scanned specimen. At a sampling distance of $80 \mathrm{~nm}$, the images cover an area of $8 \times 8 \mu \mathrm{m}^{2}$ in the focal plane.

From these double-helix images, the specimen image at a particular axial plane is constructed by "pinholing" each of the 10,000 images. This is done by integrating the products of each raw image $R_{i, j}(x, y)$ with a specific synthetic pinhole function $\mathrm{SP}_{m}(x, y)$, which can be a simple binary function (resembling a real physical pinhole), but can in general be chosen arbitrarily. Pinholing with function $\mathrm{SP}_{m}$ delivers 10,000 numbers, which represent the pixel values of one constructed $\mathrm{RESCH}$ image $I_{m}(i, j)$ :

$$
I_{m}(i, j)=\sum_{x, y} R_{i, j}(x, y) \cdot \mathrm{SP}_{m}(x, y)
$$

Our pinhole functions are binary and consist of two circular disks, the positions of which have been determined by fitting Gaussians to the two lobes of experimentally recorded doublehelix images. For this sake, a widefield z-stack of a bright fluorescent bead $\left(100 \mathrm{~nm}\right.$, dark red stained, from the TetraSpeck ${ }^{\mathrm{TM}}$ Fluorescent Microspheres Sampler Kit) was recorded with the $\mathrm{DH}$ mask in the emission path of the microscope. Note that such a widefield stack shows the emission PSF of the RESCH microscope. The synthetic pinhole functions are depicted in Fig. S1b. For the data evaluation presented in this manuscript we use three different pinhole functions, characterized by corresponding indices $m=-1,0,1$, which resulted from fitting discs to widefield DH images defocused by $-800 \mathrm{~nm}, 0$ and $+800 \mathrm{~nm}$. Using them in the pinholing process oulined in Eq. S1 results in three different images, $I_{-1}, I_{0}$ and $I_{+1}$, which show the specimen defocused by approximately $-200 \mathrm{~nm}, 0$ and $+200 \mathrm{~nm}$. Interestingly, the actually achieved defocus values are significantly smaller than $\pm 800 \mathrm{~nm}$. This is caused by the influence of the excitation PSF, which rapidly drops off at higher z-values and thus reduces the axial shift for the total PSF. 


\section{Data Processing}

(a) raw images $R_{i, j}(x, y)$
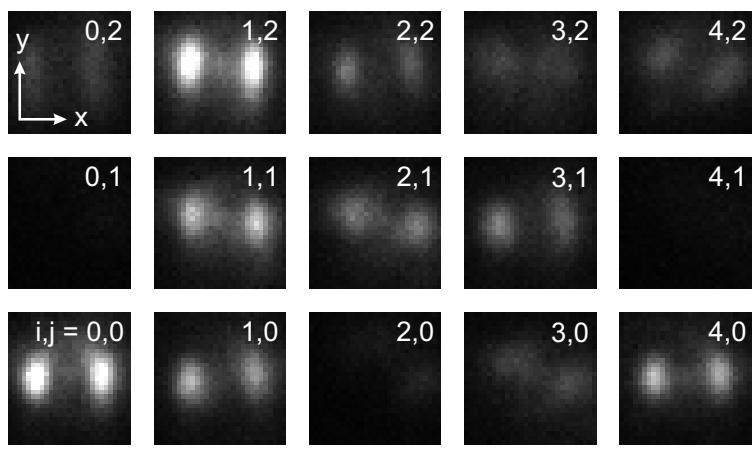

(b) pinholing $I_{m}(i, j)=\sum_{x, y} R_{i, j}(x, y) \cdot \mathrm{SP}_{m}(x, y)$
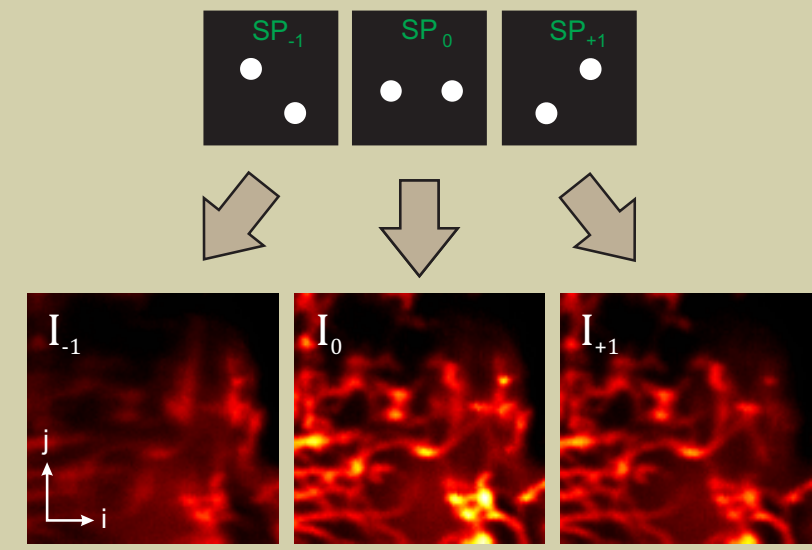

Fig. S1. RESCH data acquisition and processing; (a) raw data: one small image is taken at every sampling point $(i, j)$ in the specimen. The raw data may consist of several thousand of these images; (b) pinholing: individual axial planes are constructed from the raw data by "pinholing" with corresponding synthetic pinholes. 